\title{
EDUARDO BOSCÁ CASANOVES Y LA RENOVACIÓN TAXONÓMICA DE LOS CATÁLOGOS FAUNÍSTICOS EN ESPAÑA DURANTE EL ÚLTIMO TERCIO DEL SIGLO XIX*
}

\author{
Juanma Sánchez Arteaga
}

Dpto. de Historia de la Ciencia, IH, CSIC

\section{RESUMEN}

Con la obra del valenciano Eduardo Boscá y Casanoves, nuestra herpetología pasa de presentar sus tradicionales catálogos de Faunas locales a incluir todo un rico conjunto de indicaciones ecológicas, embriológicas y biogeográficas. Además, se incorporan criterios innovadores como el estudio in vivo, hibridaciones artificiales, etc. En todos estos cambios, la idea de la filiación genealógica de las especies aparece de forma recurrente.

PALABRAS CLAVE: Boscá, herpetología, catálogos faunísticos, transformismo, España, siglo XIX.

\section{SUMMARY}

With Eduardo Boscá y Casanoves, Spanish herpetology changes its traditional form of presenting faunistic catalogues as lists local Faunas, and begins to include a rich set of ecological, biogeographical and embryological aspects. In addition, the new catalogues include some innovating criteria such as the study in vivo of the specimens, artificial hibridations, etc. In all these changes, the idea of an evolutionary connection of the species studied, appears as the essential ideological background.

KEY WORDS: Boscá, herpetology, faunistic catalogues, transformism, Spain, $19^{\text {th }}$ century.

\section{INTRODUCCIÓN: EL LENGUAJE, EL TRABAJO Y LA REALIDAD}

Terminando el siglo XIX — como sigue sucediendo hoy en día en muchas de las actuales ramas de las ciencias naturales - , la forma final de la producción científica de los naturalistas españoles era la de textos, (científicos, claro,

\footnotetext{
* Proyecto de investigación BHA2003-01429.
} 
pero textos al fin). Desde comienzo de los años setenta de dicha centuria surgió entre la comunidad científica más avanzada del país, la urgente necesidad de crear mecanismos de salida para esos trabajos. Fue éste, precisamente, uno de los factores de mayor trascendencia en la creación más o menos contemporánea, durante esa década del siglo antepasado, de sociedades científicas que canalizasen toda esa producción a través de nuevas revistas especializadas, como las Memorias del Ateneo Propagador de las Ciencias Naturales, y, sobre todo, los Anales de la, más influyente, Sociedad Española de Historia Natural $(A S E H N)$.

El presente artículo utiliza la figura del naturalista valenciano Eduardo Boscá y Casanoves (1844-1924) para analizar la estructura interna de los textos científicos sobre biología descriptiva del último tercio del siglo XIX, que en forma de monografías y de catálogos regionales y nacionales, comenzaban a reflejar en España todo un conjunto de innovaciones y rupturas con las viejas formas de hacer historia natural hasta entonces comunes en nuestro país. A su vez, estos cambios en el lenguaje y en la estructura de la producción científica, que, como veremos, eran la manifestación de toda una constelación paralela de cambios en la ideología científica de la época, se traducirán en el descubrimiento de nuevas realidades en la naturaleza. Sobre todo, en el caso de la herpetología - y más en general, de la botánica y la zoología - esas nuevas realidades naturales se traducen en el descubrimiento de nuevas especies, nuevas formas de organismos, que el anterior modo de comprender lo natural no habría podido discriminar.

Será principalmente la incorporación del transformismo, con su idea fundamental de la filiación genealógica de los grupos de organismos afines, y a través de la introducción de nuevas dimensiones en la morfología de los organismos - en especial, relativas a los cambios que produce el tiempo-, la que posibilitará tales innovaciones y descubrimientos ${ }^{1}$. En nuestro análisis de los cambios históricos que sufre la historia natural española en el periodo de finales de siglo XIX, se ha considerado que tal transformación de la historia natural tiene un carácter inevitablemente complejo, que afecta a distintos órdenes de la realidad. Las novedades oscilan con una dualidad perfecta entre la dimensión del lenguaje de los naturalistas, es decir, de lo mental, y la dimensión de lo real, de los objetos naturales, en el tratamiento de la naturaleza por parte de los científicos. Pero

1 Sobre estos aspectos del evolucionismo español decimonónico puede consultarse el trabajo de FrAGA, X. A. (2002), «La recepción del darwinismo por los naturalistas españoles del siglo XIX: un análisis general». En: PUIG-SAMPER, M.A.; R. RUIZ y A. GALERA (eds.), Evolucionismo y Cultura. Darwinismo en España e Iberoamerica, Madrid, Doce Calles, pp. 249-265. 
a su vez, tales cambios especulares sólo se hacen posible a través de las nuevas prácticas de trabajo introducidas por los naturalistas del periodo.

Por su parte, el creciente prestigio adquirido por el experimentalismo, propiciado sobre todo por sus éxitos contundentes en otras ramas de las ciencias naturales, hasta entonces bien demarcadas de la historia natural descriptiva, tales como la fisiología, la química, la física, etc., se introducirá paulatinamente en los dominios de la botánica y de la zoología. Acaba así de redondearse el nuevo marco ideológico que creará posibilidades de innovación, abriendo nuevas perspectivas en la exploración y explotación de la naturaleza del país.

En el presente trabajo se intentarán señalar algunos de los cambios esenciales que introducen los nuevos textos naturalistas del periodo, a través de algunos de los principales trabajos de catalogación herpetológica de Eduardo Boscá, de quien se aporta, además, una breve biografía profesional. Como siempre, por otra parte, las divisiones cronológicas en el tratamiento histórico del objeto de estudio, no responden más que al interés por delimitar conceptualmente diferentes aspectos del problema, en aras de facilitar la comprensión de un todo histórico indivisible y esencialmente complejo.

\section{CRONOLOGía PROFESIONAL DE EdUARdo BosCÁ}

Eduardo Boscá desarrolló su labor científica en múltiples facetas, a saber:

- Investigación zoológica (en especial, de índole taxonómica y herpetológica). Dentro de la herpetología, su trabajo dará como fruto la descripción de un buen número de nuevos taxones europeos, entre ellos muchos endemismos peninsulares, hasta el momento desconocidos para la ciencia;

- Catalogación faunística, estrechamente unida al capítulo anterior. Aquí se atiende, de forma absolutamente innovadora en la zoología española, a

2 Sobre la biografía de Boscá existe un excelente trabajo al que sólo he tenido acceso después de haber terminado la redacción del presente artículo. Me refiero al artículo de CATALÁ, J. I. (2004), «El desarrollo de una carrera científica en un contexto institucional precario: el caso del naturalista Eduardo Boscá Casanoves y Casanoves (1843-1924)», Cronos, vol. 7, n. 1, pp. 360. Este trabajo constituye una completa visión biográfica y bibliográfica de Boscá, y supone una referencia imprescindible para todo investigador que desee aproximarse a la obra naturalística del valenciano. En cualquier caso, puesto que el presente artículo se centra casi exclusivamente en la obra de catalogación de Boscá, los datos que se aportan aquí no son redundantes con los aportados por Catalá, sino que más bien constituyen dos enfoques complementarios acerca de la obra de uno de los naturalistas españoles más significativos de su tiempo. 
los nuevos criterios metodológicos impulsados por el estudio sobre la filiación de las especies en clave evolucionista, experimental y positivista.

- Excursionismo naturalista. Ligado a su carrera profesional ha de colocarse una infatigable pasión viajera. En sus incontables expediciones —en algunos casos, favorecidas decisivamente por el apoyo y patronazgo conjunto del estado y de algunas compañías privadas de ferrocarriles-, Boscá recogía ejemplares de especies o variedades desconocidas, al tiempo que daba un fuerte impulso para los estudios de corología en la zoología española, que por primera vez veía cómo se publicaban mapas de distribución y se definían regiones faunísticas para algunas de sus especies de vertebrados.

- Docencia. Su labor como enseñante le vinculó a numerosas instituciones, tanto públicas como privadas. Señalemos su paso por la escuela de agricultura y veterinaria de Valencia, los institutos de segunda enseñanza de Xátiva, Albacete y Ciudad Real, el Jardín Botánico y la Universidad de Valencia, la Institución de Enseñanza para Mujeres, etc.

- Coleccionismo. Desde su juventud, pero muy especialmente durante el último periodo de su vida activa, Boscá dedicará sus esfuerzos a formar colecciones de objetos naturales. Esta labor la desarrolla principalmente a través del Museo Paleontológico Rodrigo Botet de Valencia, de cuya instalación y puesta en marcha Eduardo Boscá fue el principal responsable.

- Investigación paleontológica. Labor realizada, especialmente, a partir de la llegada a Valencia de la colección Botet, que constituía uno de los más completos repertorios de paleofauna mastológica del continente americano de la época.

Desde un punto de vista cronológico, se ha dividido esta biografía en tres periodos. El primero de ellos comprende la etapa de formación de Boscá, desde su ingreso en la universidad hasta su graduación como doctor y sus primeras publicaciones y contribuciones científicas. El segundo de los periodos se extiende durante los años en que Boscá trabajó como catedrático de historia natural en diferentes centros de enseñanza secundaria del país, al tiempo que realizaba numerosas excursiones naturalistas por la Península y Baleares, y publicaba con asiduidad en los Anales de la Sociedad Española de Historia Natural. A esta etapa corresponden las principales aportaciones y descubrimientos de Boscá en lo referente a la biología y sistemática de anfibios y reptiles españoles. Es este periodo el que ha sido principalmente objeto de profundización histórica en este trabajo, y al que se refieren principalmente los distintos apartados que siguen. El tercer periodo de su biografía comprende su vuelta a Valencia como Jardinero Mayor del Jardín Botánico de 
esta ciudad, su posterior nombramiento como catedrático universitario de historia natural, y su labor en torno a la referida colección paleontológica Botet, y se extiende hasta su muerte. En este periodo, la labor científica de Boscá sufre un cambio radical en su orientación, ya que, si bien aún realiza algunas importantes contribuciones en el campo de la herpetología — entre ellas, el descubrimiento en 1916 de una nueva variedad de lagartija-, su obra se centrará ahora en la Paleontología de vertebrados y en la museística.

\section{Periodo de formación.}

Nace Boscá el doce de febrero de 1843 en San Martín de Valencia, de padres valencianos, siendo bautizado en la parroquia de San Martín Obispo y San Antonio Abad con el nombre completo de Eduardo Severino y Martín Boscá Casanoves $^{3}$. Veintitrés años más tarde, Eduardo recibe el grado de bachiller en Medicina y Cirugía en la Universidad de Valencia; más tarde obtiene la licenciatura, con la calificación de aprobado, y realiza los estudios para la licenciatura en ciencias. Ya en Madrid, en la Universidad Central comienza los cursos para el doctorado en la Facultad de Ciencias, sección de naturales ${ }^{4}$, y en 1873 obtiene el grado de doctor, superando el examen de un tribunal del que formaban parte algunos de los más insignes naturalistas españoles del momento, como Juan Vilanova y Piera, Laureano Pérez Arcas, Francisco de Paula Martínez y Sáez, y Lucas de Tornos 5 . Entonces el krausismo era el ideario dominante entre los universitarios de la Central madrileña. Ése mismo año inicia su labor docente como profesor auxiliar de fisiología e higiene veterinarias en la Escuela de Agricultura y Veterinaria de la Diputación valenciana.

Su primera contribución científica importante aparece en 1872, un año antes de conseguir el doctorado. Se trata de un trabajo sobre la micología valenciana, Memoria sobre los hongos comestibles y venenosos de la provincia de Valencia, que incluye un catálogo adjunto de los hongos de España, Portugal y Baleares, y en el que se incluían 6 especies hasta entonces desconocidas en la Península. Recibe por el mismo el primer premio con medalla de oro del

3 El acta bautismal corresponde al folio 329 del libro 32 de la citada parroquia. $C f$. Archivo General de la Administración (en adelante, AGA), caja 15408.

4 Comprendía las asignaturas de organografía, fitografía, zoología de invertebrados, zoología de vertebrados. y ampliación de mineralogía.

5 Pelayo, F. (2001), «La historia natural en las universidades periféricas durante el siglo XIX. El ejemplo de Valencia», Ingenium, 7, 313-323; p. 321. A su vez, puede consultarse el AGA, expediente personal de E. Boscá, B.M.H.M.V. 
concurso convocado por el Instituto Médico Valenciano, más el título de socio de mérito ${ }^{6}$. Se inicia así su colaboración con las instituciones científicas del momento, participando ese mismo año en la fundación del madrileño Ateneo Propagador de las Ciencias Naturales, junto a otros emprendedores jóvenes naturalistas entre los que destaca Ignacio Bolívar, con quién desde esta época y hasta el final de su vida le uniría una estrecha amistad.

En 1873 su colaboración con las instituciones se continúa con su nombramiento como socio corresponsal del Museo de Ciencias Naturales de Madrid, con motivo de la donación de una serie de objetos naturales recolectados por Boscá durante una excursión naturalista a las islas Columbretes. Presentando una colección y un catálogo de reptiles y anfibios valencianos, Boscá viajará ese mismo año a Viena para participar en la Exposición Internacional. Allí recibió un diploma de honor por su trabajo, expuesto como «material científico para la enseñanza». También en 1873, Boscá ingresa como socio en la Sociedad Española de Historia Natural (SEHN).

\section{Periodo de madurez científica.}

La docencia fue una de las facetas fundamentales de la carrera científica de Boscá. Desde 1869, es decir, aún antes de conseguir el grado de doctor y mientras preparaba estos estudios, impartía lecciones, como profesor auxiliar, en la cátedra vacante de fisiología e higiene de la Escuela de Agricultura y Veterinaria de Valencia. Continuó enseñando en esta cátedra durante cinco cursos, $\mathrm{y}$, esporádicamente, dio también lecciones de zoología como suplente en la cátedra de historia natural de la Universidad de Valencia.

En 1874 consigue por oposición el puesto de catedrático en el Instituto de segunda enseñanza de Xátiva, con un sueldo de 2500 pesetas anuales — poco más de lo que cobraba un obrero especializado de la época, es decir, una miseria-. Allí impartirá, junto a la historia natural, las lecciones de física (haciéndolo gratuitamente) cubriendo la falta de docentes que el centro padecía en esta asignatura. Este mismo año aparece publicada en el Resumen de los trabajos del Ateneo Propagador de las Ciencias Naturales una memoria de Boscá sobre la recolección de reptiles, peces, moluscos y zoófitos? ${ }^{7}$.

6 BoscÁ, E. (1873), Memoria sobre los hongos comestibles y venenosos de la provincia de Valencia, Valencia, Instituto Médico Valenciano, tomo XI.

7 BosCÁ (1874), «Memoria sobre la recolección de reptiles, peces y zoófitos», Resumen de los trabajos del Ateneo Propagador de las Ciencias Naturales, Madrid. 
Desde Xátiva pasa al instituto de Albacete en 1876, y luego al de Ciudad Real, donde coincide con Enrique Serrano Fatigati, que era profesor numerario de física y química. Entre los dos naturalistas crearon un pequeño jardín botánico y ampliaron notablemente las colecciones científicas del centro, mereciendo algunos de estos objetos ser presentados como material de enseñanza en la Exposición internacional de París. En 1877, el rector del distrito universitario de Madrid nombra a estos dos naturalistas para que, en comisión de servicio, realicen un estudio naturalístico de las cuencas mineras de Almadén, Espiel y Belmar. Los materiales recogidos, minerales, rocas y fósiles, pasanron a enriquecer las colecciones de historia natural de los institutos del distrito: Toledo, Segovia, Guadalajara y Cuenca. Cuatro años más tarde, por Real Orden del 11 de Agosto de 1881, el gobierno encarga a Boscá que durante siete meses realice estudios de la herpetofauna del sur de España, debiendo presentar al ministerio presente una memoria con los resultados obtenidos.

En torno a estas fechas las instituciones comienzan a reconocer su labor investigadora, es nombrado académico de la Real Academia de Ciencias Naturales y Artes de Barcelona (1879); luego académico corresponsal nacional de la Real Academia de Ciencias Exactas, Físicas y Naturales (1882); y más tarde caballero de la Real y distinguida Orden de Carlos III (1883), por ejemplo. Y su hoja de servicios refleja su prestigio como «profesor muy conocido en el mundo científico de España y el extranjero por sus descubrimientos y publicaciones en Historia natural $\rangle^{8}$.

Entre las aportaciones herpetológicas más importantes de Boscá correspondientes a este periodo se encuentran la primera descripción del comportamiento reproductor del anfibio urodelo Pleurodeles waltl; la primera descripción de una nueva especie de víbora, la Vipera latastei; la del nuevo anfibio Alytes cisternasii; la nueva especie de ranita Hyla perezii $(=H$. meridionalis); la nueva especie endémica de eslizón Gongylus ocellatus bedriagai (=Chalcides bedriagai); el endemismo balear Lacerta muralis pytiusensis (=Podarcis pytiusensis), etc.

Periodo valenciano.

Transcurridos siete años como catedrático de instituto, en 1883 Boscá abandona Ciudad Real para incorporarse como jardinero mayor al Botánico

8 AGA caja 15408, expediente personal de Eduardo Boscá y Casanoves. 
valenciano. Anteriormente, al fallecer su maestro Cisternas ${ }^{9}$, Boscá había opositado para la cátedra de historia natural de la Universidad de Valencia, que fue concedida a José Arévalo Baca, que se había significado por su distanciamiento de la teoría evolucionista ${ }^{10}$. Significativamente, el tribunal lo componían algunos de los más cualificados representantes del conservadurismo científico del momento, como José Solano y Eulate ${ }^{11}$, Miguel Colmeiro - también de ideas católicas y conservadoras, pero más tolerante que el anterior-, y Sandalio de Pereda - a la sazón miembro de la anquilosada Academia de Ciencias y del gobierno de la Restauración Borbónica, con el cargo de consejero de instrucción pública.

Por dictamen del Consejo de Instrucción Pública, Boscá se incorpora como jardinero mayor en el jardín botánico valenciano, que entonces estaba sufriendo remodelaciones dirigidas desde la universidad. En este periodo, y hasta su paso definitivo como catedrático de historia natural de la Universidad de Valencia, impartirá gratuitamente, en momentos de ausencia de los titulares, docencia también en las cátedras de zoología, mineralogía y botánica de la facultad de Ciencias.

Fundada en Valencia la Escuela de Comercio para Señoras - después llamada Institución de Enseñanza para la Mujer — por la Sociedad Económica de Amigos del País, es nombrado vicedirector y profesor gratuito. Para esta institución crea un museo de primeras materias con más de 1000 muestras naturalísticas. Durante estos años es nombrado vocal de tribunal de oposición a distintas universidades españolas, como Granada, Oviedo y Santiago. Se le escoge como depositario provisional de la importante colección de fósiles, proveniente de Argentina, donada al ayuntamiento valenciano en 1889 por el ingeniero levantino José Rodrigo Botet. Boscá fue nombrado primero miembro de la comisión técnica y más tarde conservador de la colección, al tiempo que continua con su labor en el Botánico, como director de paseos y arbolados.

9 Rafael Cisternas (1818-1876), aprendió con Graells en la Academia de Ciencias y Artes de Barcelona y en las escuelas de la Junta de Comercio. Ocupó el puesto de Catedrático de Historia Natural en varias universidades, entre ellas la de Valencia, donde ejerció una importante influencia sobre su discípulo Boscá.

10 Pelayo (2001), p. 320.

11 Marqués del Socorro y Conde del Carpio; geólogo, miembro fundador de la Sociedad Española de Historia Natural, donde se distinguía por su catolicismo fervoroso; miembro de la reaccionaria y antidarwinista Sociedad Aragonesa de Ciencias Naturales y de la real Orden de Isabel la Católica. Cf. MARTínez SANZ, J.L. (1981), Medio siglo de Ciencia Española: La Sociedad Española de Historia natural, Tesis Doctoral, Madrid, Univ. Complutense, p. 71. 
Por Real Orden del 11 de julio de 1892 se le nombra Catedrático de Historia natural de la Universidad de Valencia ${ }^{12}$, tras ganar el concurso después de que Augusto González de Linares, a la sazón director de la estación de Biología Marina de Santander, renunciase al puesto ${ }^{13}$. En 1905 es nombrado encargado para la nueva ubicación de la Comisión Especial de la Colección Paleontológica. Boscá busca un emplazamiento donde la colección pudiera ser mostrada al público y que cuente con instalaciones adecuadas para la práctica científica. Finalmente, la colección se emplazará en el céntrico Almudín, la vieja alhóndiga gótica de la capital valenciana. En 1906 se le concede un permiso estatal para que realice un viaje a Buenos Aires, Lisboa, Londres y París, para estudiar la fauna mastológica pampeana del Pleistoceno, con objeto de avanzar sus estudios de clasificación y descripción de las especies de la colección Botet. El viaje se realizará entre octubre de ese año y septiembre de 1907. Dos años más tarde participa en el homenaje a Darwin realizado en Valencia con ocasión del centenario del nacimiento del naturalista inglés. Junto con Boscá participan Unamuno y el darwinista valenciano Peregrín Casanova.

Boscá se retira del magisterio en 1913, a la edad de setenta años. Al respecto, había comunicado a su viejo amigo Ignacio Bolívar su intención de seguir trabajando como naturalista y de publicar una obra sobre la fauna herpetológica española. El primero de octubre de ese año, Hernández Pacheco propone a los miembros de la Sociedad Española de Historia Natural, que se tributase un homenaje con ocasión de la retirada de Boscá. La Sociedad aceptará enviar una nota de simpatía y de respeto, en nombre de todos los socios ${ }^{14}$. Boscá muere en 1924, a la edad de ochenta y un años, el Boletín de la Sociedad Española de Historia Natural recoge la necrológica del «eminente naturalista D. Eduardo Boscá, bien conocido por sus estudios erpetológicos (sic) y paleontológicos, proponiendo, y así se aprueba por unanimidad, que conste en acta el sentimiento de la Sociedad por la pérdida de uno de sus miembros más antiguos y más entusiastas» ${ }^{15}$.

Al momento de su muerte, Boscá era socio de las principales instituciones científicas del país: la Sociedad Española de Historia Natural, de la Academia

12 Salinas JaQUeS, M.A. (2000), «Un proyecto de instalación del museo paleontológico J. Rodrigo Botet de Valencia en la primera década del siglo XX», Actes de les Trovades d'Historia de la Ciència i de la Tècnica, Barcelona, SCHCT, 485-492; p. 486.

13 Se conserva una carta manuscrita de González de Linares, con fecha 29-12-1891, en la caja 32/7437 del AGA.

14 Boletín de la Real Soc. Española de Hist. Natural (en adelante BRSEHN), 1913, p. 388.

15 Nota necrológica sobre Boscá, BRSEHN, 24, 1924, p. 289. 
de Ciencias Exactas, Físicas y Naturales, de la Academia de Ciencias y Artes de Barcelona, y socio corresponsal del Museo de Ciencias Naturales de Madrid $^{16}$. Sus principales publicaciones científicas, sobre todo las de tema herpetológico, se publicaron en los Anales de la Sociedad Española de Historia Natural, la Revista de la Real Academia de Ciencias, Bulletin de la Société Zoologique de France, Revue internationale de sciences de Paris, y el Resumen de los trabajos del Ateneo Propagador de las Ciencias Naturales. También colaboró con el British Museum, la Universidad de Coimbra, la Sociedad de Instrucción de Oporto, la Institución Libre de Enseñanza, y diversos institutos provinciales e instituciones privadas, sobre todo del ámbito valenciano. Entre sus colaboradores se encuentran algunos de los principales naturalistas nacionales de la época, como Juan Vilanova y Piera, Laureano Pérez Arcas, Joaquín González Hidalgo, Francisco de Paula Martínez Sáez, Ignacio Bolívar, Francisco Quiroga, Salvador Calderón y Arana, y herpetólogos internacionales de primera línea como Ferdinand Lataste, Tourneville, G. A. Boulenger, Heron Roger, Bedriaga, Sequeira, Botta, y Lorenzo Camerano.

\section{LA RENOVACIÓN DE LOS CATÁLOGOS FAUNÍSTICOS ESPAÑOLES}

«Fuerza es de confesar que las mejores descripciones no bastan de ordinario cuando se trata de separar especies afines, como lo atestiguan los naturalistas prácticos, prefiriendo la comparación directa de los objetos entre sí a la más clara de las descripciones que, sobre exigir mucho tiempo para poderlas apreciar cual corresponde, suelen a la postre dejar no pocas dudas, sobre todo si se atiende de una manera exclusiva a las frases llamadas clásicas, que tanto seducen por su precisión y laconismo; circunstancias incompatibles por lo visto con la idea de la filiación de las especies, que necesita de un riguroso detalle descriptivo y un lenguaje no siempre acomodado a las bellezas literarias» ${ }^{17}$.

La precedente cita de Boscá resume con concisión la tesis de su renovación sistemática, a saber: el cambio ideológico subyacente en la nueva forma de hacer zoología no puede distinguirse de un cambio paralelo en el lenguaje con el que los naturalistas relacionan los objetos naturales. Este cambio, a su vez, no puede separarse de la contemplación de nuevas realidades, de nuevos fenómenos naturales, nuevas especies —en el caso del ejemplo propuesto, se

16 Pelayo (2001), p. 322.

17 BoscÁ (1879d), «Las víboras de España», Anales de la Sociedad Española de Historia Natural (en adelante ASEHN), VIII, 65-86; pp. 75-76. 
trataba del descubrimiento de una nueva especie del género Vipera, endémica de la península Ibérica-, de nuevos conocimientos sobre la biología de organismos considerados hasta entonces suficientemente estudiados. Para el observador de la naturaleza se abre, de pronto, todo un abanico de nuevas perspectivas desde las que puede extraer conclusiones inesperadas. A todo este conjunto de transformaciones paralelas en el lenguaje, la ideología, y la naturaleza, debe sumarse, de forma necesaria, un desarrollo parejo de nuevas prácticas que los investigadores introducen en su relación con el objeto de estudio. En la historia natural, ocurrirá, por ejemplo, con la utilización de instrumentos o dispositivos que sirvieran para controlar cuantitativamente las condiciones en las que tienen lugar las observaciones. También lo veremos en el caso de Boscá, cuando introduce, por ejemplo, el estudio de las especies en cautividad.

Una de las características fundamentales de los trabajos taxonómicos y le realización de catálogos del periodo en que Boscá desarrolla su labor, era su aplicación siguiendo una escala geográfica local o, a lo sumo, comarcal. El tradicional localismo de este tipo de estudios, hacía que estos se emprendieran como auténticos índices parciales de una porción mínima de la naturaleza ibérica, que luego habrían de integrarse una vez completado el estudio parcial del territorio nacional en sus tres vertientes zoológica, botánica y geológica. Pero la propia palabra índice - tal y como se utilizaba en la época, en el sentido de confeccionar los índices de fauna, flora y gea de la naturaleza española - revela otros aspectos significativos de los catálogos tradicionales. En efecto, la mayoría de estos trabajos eran meros listados de especies pertenecientes a una región particular, que cuando respondía a cierto orden taxonómico, o se incluían descripciones de nuevas formas, o poco conocidas, las descripciones se hacían de acuerdo a la tradicional fórmula linneana, con mucha frecuencia en latín, que, siguiendo el método aristotélico de la división lógica, comprendía todos los aspectos relevantes de una especie utilizando cinco tipos de predicado: la esencia, el género, la diferencia, las propiedades que dimanan de la esencia y, por último, los accidentes o variaciones bajo la que el organismo del tipo en cuestión podía ser encontrado ${ }^{18}$. La renovadora

18 Por cierto que en el sistema linneano, estas 5 características comprendidas en la descripción de toda especie, surgen como manifestación de una peculiar fijación de Linneo con esa cifra: eran 5 también los rangos taxonómicos que reconocía - clase, orden, género, especie y variedad -, que a su vez había definido en función de una supuesta organización secreta de la naturaleza en la que la mencionada cantidad tenía un significado crucial. Para demostrarlo, Linneo toma ejemplos que acercan la organización jerárquica de la naturaleza a la misma organización política y militar de la sociedad creada por los humanos. Linneo nos remite a 
taxonomía que circulaba por Europa a principios del siglo XIX - Lamarck y Cuvier, son un mínimo ejemplo - no traspasó la frontera pirenaica hasta bien avanzado el siglo. El retraso podría relacionarse con numerosos factores, pero habría que mencionar - y no precisamente como el menos importante- la deficiente red de transportes que dificultaba notablemente la relación externa de nuestros científicos, estableciéndose así una barrera que difícilmente lograban superar los contadísimos profesores pensionados por el estado para ampliar estudios en el extranjero.

En la época de Boscá, en cambio, el telegrama y, sobre todo, el ferrocarril, abrían nuevas posibilidades para la rápida comunicación, incluso para el intercambio de ejemplares, entre los naturalistas. La situación permitió, sin duda - aunque no llegaría a resolver del todo el problema - que el desfase típico entre los trabajos de los naturalistas españoles y los de, por ejemplo, Francia, disminuyera, al menos en algunos campos de la historia natural, como el de la herpetología. En este contexto, muchos de los trabajos de Boscá fueron conocidos internacionalmente, a través de prestigiosas revistas del extranjero, como el Bulletin de la Société Zoologique de France. A su vez, el naturalista valenciano supo mantener una fluida correspondencia con reputados herpetólogos europeos, como Bedriaga, Lataste, Boettger, Camerano..., con quienes intercambió ejemplares cotidianamente. Aún así, el desfase científico se mantuvo y, frecuentemente, Boscá enviará al extranjero los especímenes recolectados para su precisa determinación.

Volvamos a los catálogos faunísticos de la época y a la crítica que hace Boscá, en la cita que abre este apartado, a propósito del descubrimiento de una nueva víbora, al estilo engorroso e impreciso, y a las trabas ideológicas de la vieja taxonomía fijista que aún se practicaba en el país. A través de ella, intentaremos describir algunas de las principales innovaciones que aparecen en los nuevos textos naturalistas como reflejo de los cambios sufridos en la ideología científica que los sustentaba. Observamos que el lenguaje arcaizante con el que solía describirse la morfología de las especies en latín, en términos cualitativos, y casi nunca con mediciones cuantitativas precisas, se substituye, o complementa, por un nuevo grupo de campos semánticos, que dan una nueva dimensión al concepto de especie, más allá de la morfología, e introdu-

ejemplos de la geografía política, que distinguía reinos, cantones, provincias, territorios y distritos; también cita el caso de la organización militar, en la que se establece una jerarquía de 5 rangos, con legiones, divisiones, batallones, escuadrones y soldados individuales (véase al respecto el estupendo trabajo de ERESHEFSKY, M. (1997), «The evolution of the Linnaean Hierarchy», Biology and Philosophy, 12, pp. 494-96. 
cen nuevos problemas de definición, al tiempo que cambian el significado que hasta entonces se tenía de la forma de los organismos acentuándose la cuantificación por encima de las descripción cualitativa.

Pero, aún más importante, al abandono de lo cualitativo en favor de la precisión viene a sumarse la incorporación de nuevos campos semánticos, nuevos significados, para describir la especie. Hasta entonces la taxonomía había permanecido dormida en un sueño arcaico de rimbombantes latinajos y formas perfectas, cerradas e inmutables; ahora, nuevos tecnicismos se incorporan al vocabulario de los catálogos sistemáticos. Así, la polvorienta y apolillada esencia morfológica de las especies linneanas abre su contenido a pormenores sobre las costumbres, la distribución en la península, preferencias ecológicas, hábitat, etología, reproducción, dimorfismo sexual, y otros datos deducidos de análisis pormenorizados — como la dieta alimentaria, determinada a partir del estudio de los excrementos-.

A partir de ahora, como muestran los trabajos de Boscá, la descripción de una especie se enriquecerá con nuevos términos científicos considerados irrelevantes antes de la incorporación de la ideología transformista y experimentalista-positivista. Por supuesto, este nuevo lenguaje es sólo el reflejo lingüístico de una nueva realidad que se manifiesta a los ojos del naturalista que contempla la naturaleza a través de nuevas prácticas, abandonando su fe en la autoridad establecida por las imprecisas descripciones recogidas en los libros, y se potencia al máximo la observación directa de los ejemplares en plena naturaleza o, al menos, en las colecciones, escasas, de los museos españoles.

Pero será la observación de las especies fuera de los libros y fuera de la naturaleza, en el contexto experimental, de donde surja un nuevo flujo de información que permite al naturalista plantearse nuevas preguntas, abordar nuevos problemas, e intentar resolverlos. Es este método experimental el que reclama y práctica Boscá para renovar los estudios de historia natural realizados en España:

«Un individuo que conseguimos vivo y completamente íntegro, lo conservamos por espacio de ocho días para realizar algunos experimentos sobre el envenenamiento de algunos animales que teníamos preparados, y aún colocándolo en las circunstancias de temperatura, luz, y demás que creíamos favorables, tuvimos que resolver guardarlo en el alcohol antes de que desmereciera, sin haber tenido ocasión de verle abrir la boca ni una sola vez» ${ }^{19}$.

19 BOSCÁ (1879d), p. 83. 
Los catálogos herpetológicos de Boscá.

Durante los años 1877, 1879, y 1881, se publican en Anales de la Sociedad Española de Historia Natural tres de los principales trabajos de catalogación realizados por Boscá. El inicial es el primer catálogo herpetológico realizado en nuestro país a escala peninsular, el catálogo publicado el año 77 corresponde a la fauna herpetológica de la comarca gallega de Tuy, y el impreso en 1881 corrige y añade datos nuevos a su primer catálogo nacional, y constituye una brillante aportación, si no la principal, de Boscá a la sistemática herpetológica española. Este recorrido cronológico permite analizar sus cambios ideológicos, comprobar como el naturalista valenciano introduce sucesivamente en sus obras correcciones significativas hasta llegar al catálogo de 1881 , donde su labor sistemática adquiere plena madurez sensible a las innovaciones que en este campo modelaban la historia natural.

\section{El catálogo de 1877.}

Sólo un año más tarde de la primera publicación de Darwin en España, Boscá muestra abiertamente en la Sociedad Española de Historia Natural su adhesión temprana a las teorías evolucionistas que él, como muchos de los naturalistas españoles de la época vinculados a las nuevas tendencias de la ciencia y la cultura europea a través de Francia, prefiere denominar transformistas en vez de darwinistas. Este año presenta ante la Sociedad su «Catálogo de los Reptiles y Anfibios observados en España, Portugal e Islas Baleares» ${ }^{20}$, que marca un punto de inflexión en los trabajos sistemáticos y catalogación realizados en España hasta la época, por tratarse de la primera obra realizada por un naturalista español sobre reptiles y anfibios ibéricos a escala peninsular e incluyendo las islas Baleares. El catálogo apareció como fruto del trabajo iniciado por Boscá en 1864 bajo el estímulo de su maestro Rafael Cisternas, quien impartió la asignatura de historia natural en Universidad de Valencia entre 1861 y 1876 y fue, según Boscá, pionero en la difusión de las tesis darwinistas ${ }^{21}$.

20 BosCÁ (1877a), «Catálogo de los Reptiles y Anfibios observados en España, Portugal e Islas Baleares», ASEHN, VI, pp. 39-68.

21 Pelayo (2001), pp. 316, 319; ver también Boscá (1894), Sesión apologética dedicada a don Rafael Cisternas y Fontseré. Discurso leido en la apertura del curso de 1894 del Instituto médico valenciano, Valencia. Imp. Manuel Mufre, pp. 13-14. 
El estudio detallado de las pequeñas diferencias biológicas entre formas similares comenzaba a cobrar una importancia sin precedentes. Por ejemplo, el análisis de las diferencias graduales existentes entre miembros de especies próximas, e incluso entre individuos, considerados entonces, pertenecientes a la misma especie; diferencias que la idea de la teoría de la evolución puso en primer plano de la problemática a tratar por los naturalistas. En este sentido, ante la falta de trabajos generales en nuestro país, Boscá reconoce que «aún cuando en un trabajo de tan reducidas proporciones sea indiferente la clasificación, he creído, sin embargo, que debía seguirse alguna, habiendo optado por la del eminente naturalista C.L. Bonaparte que al par que recuerda los grandes géneros linneanos en la multiplicación de sus grupos, señala preferentemente ciertos detalles de organización que tienen gran importancia para la escuela filosófica» ${ }^{22}$, refiriéndose sin duda a la corriente transformista dentro de la historia natural. También por esta carencia investigadora precedente, Boscá se vio forzado a prevenir sobre la provisionalidad de muchas de sus conclusiones lamentándose de no haber podido estudiar, todavía, con el detalle requerido las variedades faunísticas autóctonas, dado «el gran papel que para el transformismo representan» ${ }^{23}$. El trabajo manifiesta así una voluntad explícita de renovación científica que trata de poner, de una forma moderada - sin causar demasiado estrépito en las mentes más mojigatas de zoología española, algunas de las cuales gozaban de gran influencia en el seno de la SEHN — la ciencia de la clasificación al servicio de las nuevas corrientes evolucionistas.

Otra característica del catálogo de 1877 es su espíritu nacionalista, muy común entre los naturalistas españoles de la época que veían cómo las principales obras sobre la naturaleza de la península ibérica habían sido realizadas por extranjeros y contenían numerosas imprecisiones. Así, cuando Boscá comienza a realizar sus investigaciones herpetológicas «eran muy escasos los estudios realizados en el ámbito ibérico por parte de naturalistas españoles. Gran parte de las especies de herpetos más características de la península ibérica, como son las formas endémicas, habían sido descubiertas por naturalistas extranjeros: Chioglossa lusitanica por Barboza du Bocage, portugués, en 1864; Podarcis hispanica por Steindachner, austríaco, en 1870; Lacerta schreiberi por Bedriaga, alemán, en 1878; Triturus boscai ${ }^{24}$, por Fernand Lataste, francés, en 1879; Rana iberica por G. A. Boulenger, belga, también en 1879 , etc.» ${ }^{25}$.

22 Boscá (1877a), p. 42. Se refiere a la obra de Bonaparte, C. L. (1838), Amphibia europaea, Turín.

23 Ibidem (1877a), p. 39.

24 Especie nombrada así en honor de Eduardo Boscá.

25 Galán, P. (2001), «Las investigaciones herpetológicas de Seoane», Ingenium, 7, p. 256. 
En este contexto, se percibe en Boscá la influencia del catedrático de zoología Laureano Pérez Arcas, que en 1872 publicaba en los Anales de la Sociedad un trabajo sobre «Especies nuevas o críticas de la fauna Española ${ }^{26}$. Con este artículo pretendía comenzar una serie referente «a diversas especies de animales de nuestra península, dando a conocer las desconocidas, rectificando la sinonimia de otras, o reivindicando para naturalistas españoles el honor de su descubrimiento» ${ }^{27}$. Pérez Arcas se centra aquí, casi exclusivamente, en la entomofauna, particularmente en los coleópteros de la penínsu$1 \mathrm{a}^{28}$, refiriendo sobre reptiles y anfibios un único grupo controvertido, el escíndido Gongilus ocellatus ${ }^{29}$ descrito por el herpetólogo alemán Bedriaga como especie nueva para Europa. Pérez Arcas, sin embargo, atribuye a Machado (el abuelo de los poetas Antonio y Manuel) y a Boscá las primeras referencias a este grupo. Influenciado por este modo reivindicativo de Pérez Arcas, Boscá afirma haber incluido en su catálogo especies litigiosas o críticas, y publicado su trabajo para «fomentar el aumento de las especies del país $\rangle^{30}$. No obstante las intenciones patrióticas que le guían en la elaboración de su catálogo, desavenencias personales con Víctor López Seoane, el otro gran herpetólogo español del periodo, frustraron una colaboración científica provechosa convirtiendo la relación en hostilidad firme y sin disimulos.

Así se desprende de las críticas que, respaldado su amigo por Fernand Lataste, figura prominente de la herpetología francesa y europea, Boscá realizó en sus publicaciones sobre la obra del naturalista gallego, animadversión fehaciente en la correspondencia que los dos herpetólogos mantuvieron desde este año 1877 hasta 1879. Relación epistolar que con el tiempo abandona la fase de amistad formal, aunque sin colaboración científica, para llegar al enfrentamiento ${ }^{31}$.

26 PÉREZ ARCAS (1872), «Especies nuevas o críticas de la fauna española», ASEHN, I, p. 89.

27 PÉREZ ARCAS (1872), p. 89.

28 Precisamente una de las especies tratadas, Timarca splendida, había sido estudiada a partir de ejemplares colectados por Boscá en Valencia

29 Hoy conocido con el nombre específico de Chalcides bedriagai, llamado así en honor del gran herpetólogo alemán Bedriaga, con quien nos encontraremos en repetidas ocasiones a lo largo de este trabajo. Dicha especie es también conocida con el nombre común de «Eslizón ibérico». Si las reclamaciones de Pérez Arcas fueran justas - cosa que no hemos tenido ocasión de comprobar- quizá entonces, de acuerdo con las reglas de nomenclatura, esta especie debería llevar otro nombre científico, tal vez Chalcides machadoi, o bien Ch. boscai.

30 Boscá (1877a), «Catálogo de los Reptiles y Anfibios observados en España, Portugal e Islas Baleares», ASEHN, VI, 39-68; p. 39.

31 Fraga VÁzQuez, X. A. (1992), Victor López Seoane, Instituto José Cornide, La Coruña, p. 16. 
El mismo año 1877 Seoane publica en los Anales de la SEHN, anticipándose a la obra de Boscá, un "Catálogo de Anfibios y reptiles de Galicia». El trabajo mereció duras críticas por parte de Lataste que en 1878 publicaba, probablemente estimulado por Eduardo, un artículo titulado «Les reptiles de la Galicie»>32, donde el naturalista francés se sirve del catálogo de Seoane ${ }^{33}$ para hacer una crítica metodológica de los viejos catálogos naturalísticos basados en una taxonomía descriptiva y cualitativa, olvidando la filiación de las especies para fijar los grupos naturales. En este sentido, el catálogo de Boscá se redacta con la intención de corregir las graves deficiencias que caracterizaban los trabajos precedentes, como los del propio Seoane mucho menos rupturistas, sin duda, en la concepción sistemática de las especies.

Boscá había viajado ese mismo año a Lisboa, y su visita propiciará, en forma de notas publicadas por la SEHN en sus Anales, algunas reflexiones muy significativas sobre los cambios que experimentaba la biología descriptiva en la España. En la primera de sus notas da cuenta de una anomalía observa$\mathrm{da}$, de forma absolutamente fortuita, en algunos ejemplares de moluscos pertenecientes a la especie Ranella gigantea, encontrada «en una visita hecha en Mayo último a las pescaderías de Lisboa» ${ }^{34}$. Boscá fijo su atención en la anomalía de la concha, cuya morfología se aproximaba mucho a la del vecino género Triton $^{35}$. Su interés principal era señalar la importancia y necesidad de conocer la variabilidad surgida en el seno de una misma especie, variabilidad intraespecífica, para determinar categorías taxonómicas. Atendiendo en exclusiva a características morfológicas - como era habitual en los catálogos publicados por entonces en España-, podía llegarse fácilmente a confusiones sistemáticas y a la creación, en consecuencia, de taxones artificiales. Al respecto, afirma Boscá que sería deseable «que se reunieran en colección pública estos casos de diferencias extremas en las formas específicas de los seres, para formar conceptos definitivos sobre la Historia Natural propiamente dicha» ${ }^{36}$.

La segunda nota, en estrecha relación con la anterior, se refiere a las modificaciones taxonómicas necesarias para corregir el genero Rana, algunos de

32 Lataste, F. (1878), «Les reptiles de la Galicie», Revue internationale des Sciences, 22, pp. 693-697.

33 Parece ser que la habilidad de Seoane como sistemático de herpetofauna aún no estaba muy madurada. Según su principal biógrafo, de los 40 taxones por él estudiados, en total hay doce descritos de forma confusa y otros cinco que, sencillamente, están mal determinados. Véase FRAGA VÁZQUEZ (1992), p. 16.

34 BoSCÁ (1878a), «Anomalía de una Ranella gigantea», ASEHN, VII, 1878, 25.

35 Ibidem.

36 Ibidem, p. 26. 
cuyos especímenes habían sido erróneamente clasificados en su propio catálogo de 1877. Por error Boscá había incluido algunos ejemplares de la especie agilis dentro de la especie Rana temporaria atendiendo tan sólo a criterios morfológicos. Las correcciones evidencian la voluntad reformadora que asiste a Boscá en términos de sistemática, gobernada ahora por el principio de la "filiación de las especies (...) la idea más científica a la que se puede aspirar en la parte descriptiva» ${ }^{37}$.

\section{El catálogo herpetológico de la región de Tuy (1879).}

El nuevo catálogo fue el resultado de la excursión herpetológica realizada por Boscá al monte gallego de San Julián de Tuy el año 1879. Se debe ubicar, por tanto, en el contexto de las críticas vertidas el año anterior por Lataste hacia el trabajo de Seoane sobre los reptiles y anfibios de Galicia. No sorprende, pues, que uno de los primeros objetivos científicos del viaje sea la ampliación y corrección del catálogo del naturalista gallego, estereotipo, como hemos visto, de la clase de trabajo sistemático a superar. Boscá confiesa que tuvo presente la obra de Seoane al redactar su propio catálogo para mejorar y «completar en lo posible las especies citadas por el ya dicho Sr. Seoane en sus Reptiles y anfibios de Galicia» ${ }^{38}$. Tampoco es de extrañar que Seoane ${ }^{39}$ no tomase con agrado la iniciativa del joven naturalista valenciano. Pero no fue sólo con Boscá con quien el carismático gallego mantuvo importantes polémicas a lo largo de su carrera científica ${ }^{40}$. Por ejemplo, también en 1879 Seoane mantuvo un duro enfrentamiento con otro miembro de la SEHN, Macho de Velado, discutiendo sobre los Moluscos de agua dulce de Galicia. Como era costumbre en Seoane - que por su lejanía no acudía a las sesiones de la SEHN en Madrid- envió una nota sobre el tema a la comisión de publicación de la Sociedad para su difusión entre los socios. De la reunión man-

37 BoSCÁ (1878b), «Nota sobre Rana temporaria/ R. Agilis», ASEHN, VII, pp. 29-30.

38 BosCÁ (1879), «Nota herpetológica. Una excursión hecha en el monte San Julián de Tuy», ASEHN, VIII, 463-484; p. 483.

39 Para todo lo que concierne a Seoane, el investigador más competente es sin duda Fraga Vázquez, y sus trabajos son una fecunda referencia para cualquiera que se interese en la figura del naturalista gallego.

40 Por otra parte, Seoane no estaba sólo en esta peculiaridad: ya sabemos del concepto en que el eminente Graells tenía de algunos fundadores de la SEHN. Sin duda, toda la historia (y el presente) de la Ciencia está plagada de numerosos casos en los que una cierta antipatía profesional es llevada más allá de lo estrictamente científico. 
tenida por la comisión para estudiar la petición se deduce el tono agrio y personal de la nota, cuya publicación en los Anales fue desestimada «con el fin de que, suprimiendo todo lo que no correspondiese a una verdadera réplica científica, se evitase la publicación en los ANALES de escritos que, siendo más o menos personales no la interesan, y que pueden herir el amor propio de los socios, y en este caso, de los señores Seoane y Macho de Velado, que vienen dando pruebas de entusiasmo científico, siendo de esperar que acaso se pudiesen restablecer las buenas relaciones entre ambos» $\rangle^{41}$.

Entre las principales menciones realizadas por Boscá al catálogo de Seoane se encuentran algunas adiciones y, principalmente, numerosas correcciones. Como referencias, cabe destacar al mencionado Gongylus ocelatus ${ }^{42}$, que el gallego sólo había podido citar con dudas «en su interesante catálogo» ${ }^{43}$; y la variedad sardous del «sapillo pintojo». Mayor espacio ocupan las referencias puramente correctoras que a veces tiene un tono irónico, incluso de mofa: «no pude menos de extrañar que el Sr. Seoane nada diga de la Zamenis riccioli, en su ya citado catálogo, cuando precisamente nos habla de los alrededores de Tuy, en donde no dudo en calificar como abundante la dicha especie. Habla en cambio del Zacholus austriacus Laur., del que no he podido encontrar ni un solo individuo» ${ }^{44}$. No había ni rastro del animal en los lugares citados por Seoane, y su descripción de los hábitos de la especie merecía poca confianza: «por cierto que le asigna como vivienda los tejados de las casas y en los corrales, lo que parece poco conforme con lo que de ella dice el reputado profesor Sr. Graells» ${ }^{45}$. Graells calificó al reptil como una especie de montaña, por lo cual era poco probable que habitara en Tuy, situada en una zona de colinas suaves y cercana al mar. Boscá, que en otras ocasiones no daba excesivo crédito a los viejos trabajos de zoología, en esta ocasión, interesadamente, utiliza las autoridades para apoyar su juicio contra el rival. Pero su juicio comparativo frente a la colección herpetológica del Museo universitario de Coimbra es mas riguroso. Aquí halló Boscá la práctica totalidad de los reptiles del norte de Portugal no encontrándose la citada especie, y, con o

41 Nota en $A S E H N, 1879$, p. 4.

42 Recordemos: el G. ocellatus o Eslizón ibérico, hoy denominado por los zoólogos con el nombre de Chalcides bedriagai, considerada ya como una especie polémica —o «critica»por Pérez Arcas (ver nota 26).

43 Boscá (1879), p. 475.

44 Ibidem p. 476.

45 Ibidem. 
sin ironía, afirma que «pudiera tratarse de algunas de las dos especies únicas que faltaban en aquella colección local, según me aseguraron» ${ }^{46}$.

Junto a la polémica científica el viaje a Tuy servirá para obtener nuevos datos sobre la herpetología española, incluyendo un buen número de especies: Thimon ocellatus (Lacerta ocellata Daud) o sardón; Podarcis muralis (Seps muralis Laur) o lagarta; Gongylus ocellatus (Lacerta ocellata Forskal) o Licrán; Zamenis riccioli (Coluber riccioli Metax) o cobra; Natrix viperina (Coluber viperinus Latr) o cobra, Rana esculenta L., también rana, o culler (las larvas se llaman cagotes); Rana iberica Boulanger; Pelobates cultipres (Rana cultipres Cuv.) o sapo; Discoglossus pictus Otth.; Alytes obstetricans var Boscai Lataste, o sapiño; Bufo calamita Laur., o sapiño; Salamandra maculosa (Lacerta salamandra L.), Chioglossa lusitanicum Barbosa, o saramajanta; Pelonectes boscai Lataste, o saramajanta ${ }^{47}$.

La novedad más interesante desde el punto de vista de los cambios introducidos en las obras de catalogación es la información biológica que sobre cada especie se incluye en su descripción; por ejemplo, se informa sobre la denominación local, el hábitat, el dimorfismo sexual, las variaciones en edad, en color, sobre formas intermedias, variedades geográficas, etc. Conjunto informativo ciertamente novedoso para la herpetología nacional. Veamos un ejemplo. Algunas de las especies descritas eran codiciadas por Boscá desde hacía tiempo, como la salamandra rabilarga (Chioglossa lusitanica). De Chioglossa, especie enigmática y conocida desde hacía pocos años, se dan apuntes sobre localización, comportamiento, hábitos, movilidad, preferencias subterráneas, alimentación (datos obtenidos mediante la disección y el estudio del contenido estomacal), sobre su técnica de ocultación bajo el musgo, etc. El catálogo incluye también especies hasta entonces desconocidas como el Alytes obstetricans var

46 Boscá (1879), p. 477. Hoy sabemos que el área de distribución de la especie comprende tanto el sur de Galicia como todo el norte de Portugal. Y si bien es cierto que el animal puede alcanzar en montaña cotas de hasta 1100 metros de altura, se le puede encontrar también cerca de las costas, en terrenos y climas muy variables; todo lo cuál parece dar la razón a Seoane en este caso. De cualquier modo, de quién era la razón es intrascendente para los objetivos del presente trabajo. En otras controversias entre Seoane y Boscá será éste último quien - a los ojos de nuestro conocimiento presente- acierte. Nos interesa, más que desfacer entuertos (en este caso, históricos), más que exponer cómo se resolvieron estos, comprender, en cambio, cómo se llegan a configurar esas problemáticas, y cómo en el proceso hay factores racionales e irracionales intrínsecamente ligados.

47 Debido a no haber tenido la ocasión de contrastar todos los nombres científicos con los aceptados actualmente, mantenemos la nomenclatura decimonónica de las especies, a pesar de que el nombre de muchas de ellas ha cambiado en nuestros días. 
Boscai Lataste, variedad de sapillo partero estudiada por Lataste a partir de ejemplares recolectados por Boscá en las inmediaciones de Valencia.

Otro de los resultados científicos interesantes del viaje fue la corrección del catálogo precedente sobre los reptiles y anfibios de la península. Antes Boscá, siguiendo la obra de C. L. Bonaparte Amphibia europaea, situaba la especie de anfibio Pelobates fuscus dentro de la península, Ahora, en cambio, se corrige a Bonaparte que se había confundido con la especie Pelobates cultipres, sapo de espuelas, único miembro del género habitante en este área.

Regeneracionismo científico. Correcciones y adicciones al catálogo herpetológico español (1881).

El catálogo de $1881^{48}$ se puede, sin duda, calificar como la principal aportación de Boscá, y probablemente de la herpetología española decimonónica, a la biología descriptiva, la taxonomía evolucionista y a la corología de vertebrados no fósiles; estudio reconocido internacionalmente con su publicación simultánea en francés en el Bulletin de la Société Zoologique de France ${ }^{49}$. La obra surge de la mano de un Boscá en plena madurez que compendia sus anteriores esfuerzos por superar prácticas obsoletas incorporando los nuevos métodos experimentales que posibilitan la cría en cautividad para el estudio de animales in vivo, el análisis del desarrollo embriológico, y el estudio de numerosos organismos de cada taxón, por ejemplo; sin olvidar las facilidades que los nuevos medios de comunicación ofrecen para el trabajo de campo en áreas de distribución alejadas, y conformar el primer y más importante trabajo español del siglo sobre biogeografía de vertebrados. En este capítulo, de forma absolutamente pionera en nuestro país, su catálogo de fauna peninsular e isleña incorpora el primer mapa de distribución geográfica de anfibios y reptiles con un total de 18 familias, 39 géneros y 55 «formas» ${ }^{50}$, entre especies y

48 BosCÁ (1881a), «Correcciones y adicciones al catálogo de los reptiles y anfibios de España, Portugal e Islas Baleares, seguido de un resumen general sobre su distribución en la península», $A S E H N$, X, pp. 89-112.

49 BoscÁ (1881b), «Catalogue des reptiles et amphibiens de la Péninsule Ibérique et des Iles Baléares», separata Bull. Soc. Zool. France, pp. 1-48.

50 Boscá emplea deliberadamente esta voz, en lugar de la palabra «especie», con la intención de hacer patente la crisis de los viejos conceptos sistemáticos ante las nuevas perspectivas abiertas sobre estos por el evolucionismo. 
subespecies. Así, el particular tipo de texto científico de los catálogos naturalistas incluía gráficos, un adorno que en el futuro será predominante.

11 nuevas especies recogía el catálogo, y un total de 3 géneros y hasta 8 especies o subespecies endémicas, exclusivas, de la fauna peninsular e insular, «al menos por ahora» ${ }^{51}$. La lista completa de novedades para la fauna y la ciencia españolas era:

1 Lacerta schreiberi

2 Psamodromus cinereus Bonap

3 Gongylus ocelatus Bedriagai nobis

4 Vipera berus Seoanei Lataste

5 Vipera latastei nobis

6 Rana iberica Boulenger

7 Rana fusca Rösel

8 Hyla Perezzi nobis

9 Ammorictys Cisternassi nobis ${ }^{52}$

10 Alytes obstetricans Boscai

11 Pelonectes Boscai

Otras especies incluidas anteriormente por error ahora se corregían: la $\mathrm{Vi}$ pera ammodytes, que había sido confundida con la nueva especie $V$. Latastei; el Pelobates fuscus, especie del norte de Europa y que sin embargo había sido citada en la península por Bonaparte, confundiéndolo con P. Cultipres; el Discoglossus sardous (en realidad se trataba de una variedad del D. pictus); el Triton parisinus, confundido por Seoane con el Pelonectes Boscai. Además, el catálogo aborda de forma explícita el problema de la variabilidad subespecífica en el reino animal superando todos los titubeos que la académica biología española mantuvo frente al transformismo, exponiéndose la importancia de la nueva taxonomía, la corología y los estudios embriológicos para el estudio del origen de las especies ${ }^{53}$. La herpetofauna se acomodaba fácilmente al planteamiento transformista, pues en anfibios y reptiles existían un buen número de casos de «especies polimorfas» equivocadamente catalogados por la sistemática linneana como grupos específicos diferentes (por ejemplo, el cita-

51 BosCÁ (1881a), p. 90.

52 La creación de este nuevo género, sinónimo de Alytes, sigue el criterio recientemente expresado por LATASTE (1879b), «Sur une nouvelle forme de batracien anoure d'Éurope», Compt. Rend. Acad. Des Sciences, p. 983.

53 Boscá (1881a), p. 100. 
do caso de los Discoglossus sardous, y la variedad marroquí D. scovazii, incluidos ambos ahora dentro de la especie $D$. pictus).

También se dio el caso contrario, especies distintas se incluyeron erróneamente bajo la misma denominación por incapacidad para comprender el valor sistemático de factores como la corología (distribución geográfica), o las fases estacionales del ciclo vital de la especie. Este, por ejemplo, fue el caso del género Rana. En mayo de 1877 Boscá viajó a Lisboa visitando el Museo de la Escuela Politécnica. Aquí coincidió con dos relevantes herpetólogos de la época, el portugués Barbosa du Bocage ${ }^{54}$ y el francés Fernand Lataste. De sus conversaciones Boscá dedujo que algunos ejemplares adscritos a Rana temporaria en su Catálogo de 1877, concretamente los de Guadarrama y Santander, pertenecían a la especie Rana agilis, todavía no descrita para la Península. En realidad correspondían $R$. Iberica, como veremos a continuación. Ambas especies podían distinguirse con menor dificultad durante la época de reproducción, y la vieja sistemática no había sabido prestar atención a tales variaciones.

Anteriormente a las clarificaciones que el herpetólogo belga G.A. Boulenger había introducido en la sistemática del género que nos ocupa, formaban parte de $R$. temporaria un total de cinco especies que luego serían bien diferenciadas. En el catálogo de 1881, por el contrario, se sabe ya que, «Como en muchas de las grandes especies de Linneo, los caracteres correspondientes han pasado a formar el distintivo de un grupo del género Rana, en el que se afilian las formas europeas $R$. fusca Rösel, $R$.. arvalis Nils, $R$. iberica Boulenger, $R$. Latastei Boulenger, y $R$. agilis Thomas ${ }^{55}$. Ahora, además, Boscá estaba en condiciones de estipular que «de las cinco especies dichas corresponden a nuestra península la primera y la tercera; y nos permitimos suponer que las indicaciones hechas sobre la $R$. temporaria en España y Portugal (sic) serán con referencia a la $R$. iberica, pues la $R$. fusca no se ha encontrado ahora más que en las montañas de Galicia» ${ }^{56}$.

Estas puntualizaciones resultaban de gran importancia pues, a través de ellas, se introducía en nuestra zoología el concepto de grupo natural ${ }^{57}$, que

54 Quien, entre otros muchos méritos, tenía el de haber descubierto uno de los más famosos endemismos de la fauna ibérica, la «salamandra rabilarga» o Chioglossa lusitanica.

55 BosCÁ (1881a), p. 91. Conviene subrayar el significado puramente evolucionista de la expresión se afilian, que remite a la genealogía, y las especies que se desarrollan de forma genealógica son especies que se transforman a lo largo de la evolución orgánica.

56 BoscÁ (1881a), p. 92.

57 Lo que los biólogos evolucionistas actuales denominan grupo monofilético, es decir, que cuenta con un origen evolutivo común a partir de una especie ancestral. 
designaba al conjunto de organismos con un origen genealógico común, es decir, que han evolucionado a partir de un tronco específico primitivo. Al aplicar este nuevo concepto a los grupos herpetológicos el resultado era revolucionario al contar con la filiación como el fundamento básico de sus categorías clasificatorias. Así, el conjunto de los ejemplares asignados a la especie Rana temporaria, que había sido descrita exclusivamente a partir de rasgos morfológicos ${ }^{58}$, realmente incluía un grupo natural de especies estrechamente emparentadas. Para Boscá, los nuevos criterios aplicados por los sistemáticos modernos obligaba a revisar todas las colecciones de reptiles del país, y sólo «el exagerado apego a determinados escritos autoritarios, en asuntos en que no cabe más que el ver las cosas (...) ha sostenido por mucho tiempo equivocaciones de otro modo inconcebibles ${ }^{59}$.

Otro ejemplo fue el caso de las lagartijas de los muros. Sobre ellas, un atento estudio de su polimorfismo cambió radicalmente la clasificación, y fue el especialista alemán Bedriaga el encargado de deshacer el entuerto sistemático $^{60}$. Bedriaga dedicó su investigación a estudiar la variabilidad de la lagartija valorando la forma de las escamas, la talla y la coloración de los individuos en los distintos países europeos. Por su polimorfismo la «denominación específica de muralis $L$. es ya incompatible con el criterio contemporáneo», explicaba Boscá valorando el trabajo del naturalista alemán y asumiendo su ideario $^{61}$. El polimorfismo era un factor sistemático fundamental que los naturalistas españoles debían incorporar para remediar su retraso científico. Además, esta novedosa utilización del concepto de variabilidad resultaba muy útil para el estudio de la herpetofauna peninsular por ser abundantes los casos de especies críticas y polimorfas. Como ejemplos refería Boscá las especies Cistudo orbicularis L., Lacerta viridis L, Acanthodactylis vulgaris Dum et Bibr.; Anguis fragilis L.; Coelopeltis mospessulana Herm; Tropinodotus viperinus Latr., Rana esculenta L., Discoglossus pictus Otth; y Salamandra maculosa Laur, donde «se encuentra también la misma tendencia a la variación» ${ }^{62}$, cuyo deficiente conocimiento hacía aún más apremiante la colaboración entre

58 El principal criterio de determinación consistía en la presencia de manchas oscuras que los ejemplares de la presumida especie presentaban siempre en la zona temporal.

59 BOSCÁ (1881a), p. 90.

60 BedriagA, J. (1879), «Mémoire sur les variétés européennes du lézard des murailles», Bull. Soc. Zool. France, pp. 194-228.

61 BosCÁ (1881a), p. 100. Bedriaga dividió este grupo natural, considerado como una especie homogénea, en cuatro grupos principales con un total de 16 variedades y 18 subvariedades.

62 BosCÁ (1881a), p. 100. 
los herpetólogos españoles, y aconsejaba prudencia en su estudio: «tanto estas especies más variables, como otras que lo son menos, exigen mayor copia de datos de los que hoy poseemos, para establecer algún criterio metódico al darlos a conocer» ${ }^{63}$. La crítica científica de Boscá repercutía en el ámbito político - coincidiendo con la llegada al poder del gobierno liberal y las esperanzas reformistas que conllevaba para el entorno progresista-, lamentándose del precario estado de la investigación española, al servicio de una «ciencia especulativa», no sufragada por el estado y, por ello, muy limitada en medios respecto a los países «más cultos» ${ }^{64}$.

Si la biología descriptiva española quería aproximarse al nivel de las naciones europeas científicamente más avanzadas, era preciso realizar con urgencia trabajos de base ignorados aún en nuestro país. Por ejemplo, para realizar una biología descriptiva competente era preciso concluir antes, al menos de forma provisional, la tarea de catalogación frustrada desde los isabelinos tiempos de Graells; resolver la cuestión de las especies críticas aparcada desde los primeros intentos de Pérez Arcas por solucionarla; y generalizar los trabajos de distribución geográfica. La magnitud de la empresa hacía imposible su realización si, como hasta entonces, se emprendía de una forma aislada e individual, sin apoyo institucional ni estatal. Boscá no halla otra solución que la asociación de los distintos especialistas y, para facilitar esta colaboración, al menos en lo concerniente a la herpetología, se propone resumir en su catálogo las más recientes publicaciones extranjeras y nacionales sobre herpetofauna ibérica y balear, para llamar «la atención de nuestros naturalistas, por si gustan asociarse para la terminación de esta parte de la fauna Ibérica y de las Baleares» ${ }^{65}$. El catálogo cubre así una faceta bibliográfica también novedosa.

\section{Corología y Ecología.}

Para estudiar la variabilidad de las especies la moderna sistemática había tenido que considerar argumentos novedosos como la corología, hasta entonces prácticamente desatendidos. Al respecto, la obra de Boscá se enmarca

63 BosCÁ (1881a), p. 101.

64 refiriéndose sin duda, sobre todo, a Francia y a Alemania, a cuyos investigadores reconoce Boscá el papel de «árbitros indiscutibles» en estas cuestiones durante mucho tiempo.

65 BosCÁ (1881a). p. 90. Entre las obras de autores españoles señaladas, además de los trabajos de Seoane sobre la fauna de Galicia, cita la «Erpetologia hispalensis» (sic) de Machado para la provincia de Sevilla, y los trabajos que el profesor Barceló para la herpetología balear. 
dentro de una innovadora corriente de trabajos donde algunos de los naturalistas españoles comenzaban a tener muy en cuenta la biogeografía y la ecología para clasificar las especies. Como ejemplo podemos los trabajos de Salvador y Arana ${ }^{66}$, con algunos apuntes de paleocorología, los de Vayreda y Vila y, sobre todo, los de Lázaro Ibiza en colaboración con Tomás Andrés y Tubilla; sin olvidar a Odón de Buen ${ }^{67}$.

Por su parte, el nuevo catálogo de Boscá incorpora plenamente este criterio ecológico-corológico, siendo pionero en España para el ámbito de los vertebrados. Consideraba Bocá que tres duros años de investigación, sumado a su precedentes estudios, «permiten fijar el área que ocupan la mayor parte de las especies en nuestra península» ${ }^{68}$. Pero la peculiar biología de estos animales introducía un carácter provisional que no olvida en sus conclusiones: «tratándose de animales que ofrecen por lo común bastantes medios de resistencia para con los agentes exteriores, pocas serán las especies que se hallan hoy limitadas a tal o cual estancia obligada» ${ }^{69}$.

La distribución herpetológica realizada por Boscá para la Península y Baleares consideraba la altitud y el hábitat como los factores climáticoecológicos fundamentales. Elevadas alturas, la nieve y la humedad relativa, eran factores determinantes para establecer la presencia o ausencia de ciertas especies. Buenos indicadores de otras eran la vegetación y la existencia de microfauna alpina, así como la presencia de microclimas, es decir, «estaciones aisladas a la manera de oasis, con suficiente carácter para el fomento de determinadas especies ${ }^{70}$. Los hábitats más comunes para muchas eran las localidades pedregosas, las zonas con arena o tierra o vegetación intercalada, las zonas cultivadas, y las áreas antrópicas, indicándose que la presencia de reptiles en zonas ocupadas por humanos respondía a la desaparición de los refugios naturales habituales. La colonización de muros construidos y de zonas cultivadas responde «a la falta de mejores abrigos naturales, aunque en algún caso pudiera interpretarse como un verdadero progreso en las costum-

66 CALDERON y ARANa (1880), «Ensayos de geología general. La Evolución Terrestre», ASEHN, IX; pp. 15-47.

67 VAYREDA Y Vila (1879), «Plantas notables por su utilidad o rareza que crecen espontáneamente en Cataluña, o sea apuntes para la flora catalana», ASEHN, VIII, pp. 345-462; IBIZA, L.; ANDRÉS Y TUBILlA, T. (1881), «Revista Crítica de las Malváceas españolas», $A S E H N, \mathrm{X}$, pp. 392-428; OdÓN DE BUÉN (1883), «Apuntes Geográfico Botánicos sobre la zona central de la Península Ibérica», $A S E H N$, XII, pp. 420-440.

68 BosCÁ (1881a), p. 102.

69 Ibidem.

$70 \quad$ Ibidem, p. 103. 
bres, mediante el cual se facilita el cumplimiento de las necesidades de su alimentación $\rangle^{71}$. El cambio del paisaje natural obligaba a modificar sus costumbres a muchas especies lo que, en el ambiente lamarckiano de la época, se interpretaba como un incipiente proceso evolutivo de nuevas razas y variedades donde los rasgos de comportamiento, unidos a cambios en la morfología, resultarían determinantes para la aparición de nuevas formas orgánicas.

La consecuencia más sobresaliente de tan ingente tarea fue la elaboración del primer mapa de distribución de las especies de reptiles y anfibios del país, objetivo largamente perseguido por el herpetólogo valenciano que desde sus excursiones a Tuy no escondía su intención de formar un mapa zoológico de la península. Transcurrido este tiempo se siente capacitado para ofrecer esta imagen gráfica, distinguiendo dos claras regiones faunísticas para los herpetos ibéricos, junto a la región balear: «la distribución geográfica de los reptiles y anfibios dentro de la Península, puede fijarse en dos regiones separadas por una línea que, partiendo de los Pirineos orientales, se dirigiera a la desembocadura del Duero: haciendo una profunda inflexión sobre el tercio superior de la cuenca del Ebro, y otra curva en sentido inverso y más extensa sobre la meseta de Castilla la Vieja, alcanzando las estribaciones de la cordillera carpetana» ${ }^{72}$. Para la confección del mapa Boscá utilizó tanto los datos por él recopilados como la información aportada por naturalistas españoles solventes, como Macho Velado, Seoane, Calderón, Gogorza, Sainz y Gutiérrez, Martínez Sáez, Serrano, Mazarredo, Uhagón, Silva, Viar, Asso, Pérez Arcas, Martorell, Roca, Pérez Mínguez, Cisternas, Graells, Bolívar, Castellarnau, Breñosa, P. de Ávila, Sanz de Diego, Ribera, Antón, Guirao, y los recientes trabajos de Cardona y Barceló para las Baleares ${ }^{73}$. En cierto modo, el mapa era una imagen colectiva con la que Boscá hacía realidad, a título personal, su fórmula asociativa para el progreso de la historia natural en España.

71 Boscá (1881a), p. 103.

72 Ibidem, p. 104.

73 BARCELÓ (1876), Catálogo de los reptiles y conchas terrestres y de agua dulce de las Baleares, Palma de Mallorca. Boscá también utiliza la información del naturalista Juan Ramis, que en 1786 hablaba de un tipo de urodelo, al que denomina Lacerta aquatica como propia de les Illes (no se conocía ninguna cita posterior de estos anfibios para la fauna balear). Citamos por la edición RAMIS RAMIS, J. (1814), Specimen animalium, vegetabilium et mineralium in insula Minorica frequentiorum ad norman linneani sistematis, excudebat Magone Balearium (Mahón), Pedro Antonio Serra. 


\section{CONCLUSION}

A lo largo de estas páginas hemos analizado la labor herpetológica de Boscá desde el punto de vista taxonómico. Entre sus principales aportaciones zoológicas se encuentra la descripción de un número considerable de nuevos taxones - entre ellos, bastantes endemismos - , su estudio de la variabilidad como objeto sistemático, y la realización de mapas de distribución zoológica. Actividades desplegadas en un contexto de renovación de la historia natural acorde con los parámetros científicos establecidos para la disciplina fuera de España. Tarea donde muestra su vinculación con la ideología evolucionista, convirtiéndole en uno de los primeros naturalistas españoles en incorporar el nuevo ideario a su práctica científica cotidiana, siendo capaz de traducir la nueva filosofía en cambios metodológicos puestos en práctica en su investigación de la naturaleza. Eduardo Boscá y Casanoves se perfila en la historia de la biología española como uno de los principales responsables de la transformación, tardía, de la historia natural en la disciplina que iniciado el siglo XIX en Europa se conocía como biología. 